\title{
Krüppel-like factor 5 is upregulated and induces cell proliferation in endometrial cancer
}

\author{
TETSUYA MORITAKE $^{1 *}$, JUNYA KOJIMA $^{1 *}$, KAIYU KUBOTA $^{1,2}$, \\ FUMITOSHI TERAUCHI ${ }^{1}$, KEIICHI ISAKA ${ }^{1}$ and HIROTAKA NISHI ${ }^{1}$ \\ ${ }^{1}$ Department of Obstetrics and Gynecology, Tokyo Medical University, Shinjuku, Tokyo 160-0023; \\ ${ }^{2}$ Division of Grassland Farming, Institute of Livestock and Grassland Science, National Agriculture and \\ Food Research Organization, Nasushiobara, Tochigi 329-2793, Japan
}

Received October 9, 2020; Accepted February 22, 2021

DOI: $10.3892 / 01.2021 .12745$

\begin{abstract}
Krüppel-like factor 5 (KLF5) is involved in various cellular processes, such as cell proliferation and survival. KLF5 has been implicated in cancer pathology. The aim of the present study was to investigate the expression levels and function of KLF5 in endometrial cancer. A total of 30 patients, including 12 patients with endometrial cancer and 18 with benign gynecological diseases (controls), were enrolled at Tokyo Medical University (Tokyo, Japan) between March 2017 and May 2018. Endometrial cancer and control endometrium tissues were collected, and the expression levels of KLF5 were determined using reverse transcription-quantitative PCR, western blotting and immunohistochemistry. For the functional analyses of KLF5 in endometrial cancer, the present study employed a loss-of-function strategy in the human endometrial cancer cell lines in vitro. Ishikawa and HEC1 cells were transduced with lentiviral constructs expressing shRNAs targeting KLF5. MTT and TUNEL assays were performed in cells after knockdown to analyze the role of KLF5 in cell proliferation and survival. The results revealed that the mRNA and protein expression levels of KLF5 were increased in endometrial cancer tissues. In vitro analyses demonstrated that depletion of KLF5 inhibited cell proliferation and decreased the expression levels of cyclin E1. However, silencing KLF5 did not induce cell death. Overall, these results indicated that KLF5 may be crucial in the tumorigenesis of endometrial cancer and has potential as a therapeutic target.
\end{abstract}

Correspondence to: Dr Kaiyu Kubota, Division of Grassland Farming, Institute of Livestock and Grassland Science, National Agriculture and Food Research Organization, 768 Senbonmatsu, Nasushiobara, Tochigi 329-2793, Japan

E-mail: kubotak028@affrc.go.jp

*Contributed equally

Key words: Krüppel-like factor 5, endometrial cancer, cell proliferation, cyclin E1, oncogene

\section{Introduction}

Endometrial cancer is one of the most common gynecological tumors that has seen a dramatic increase in incidence worldwide $(1,2)$. This increase has a number of causes, including an aging population, increased calorie intake and the concomitant rise of obesity, and reproductive factors, such as early age menarche, nulliparity, late-onset menopause, and use of unopposed estrogens (3). These reproductive factors are associated with a strong risk for endometrial cancer. Postmenopausal women have the greatest incidence, but $14 \%$ of endometrial cancers are diagnosed in premenopausal women, including $5 \%$ of cases younger than 40 years old. The increased incidence and population shift to younger women are proposed to continue into the future $(3,4)$. Although patients diagnosed with early stage disease are associated with a good prognosis of endometrial cancer, patients with recurrent or metastatic disease have limited treatment options and shorter overall survival (5). Thus, understanding the pathophysiology of endometrial cancer is key to improving its diagnosis and treatment.

No targeted therapies have been approved or available for endometrial cancer. Several completed clinical trials targeted deregulated pathways, such as the PI3K/AKT/mammalian target of the rapamycin (mTOR), but to date results have been poor (3). Therefore, other potential targets need to be investigated. Krüppel-like factors (KLFs) comprise a family of zinc finger transcription factor proteins that control cell proliferation, apoptosis, migration, and differentiation (6). Multiple KLFs function as oncogenes or tumor suppressors depending on the specific tissues and cellular environments $(7,8)$. KLF5 is upregulated and functions as an oncogene in colorectal (9), cervical (10), and breast cancer (11) and downregulated and acts as a tumor suppressor in lung (12), and prostate cancer (13). Although KLF5 is expressed in non-tumor endometrial epithelial (14) and stromal cells (15), the role of KLF5 in endometrial cancer remains to be explored. Therefore, this study aims at investigating the expression and function of KLF5 in endometrial cancer.

\section{Materials and methods}

Human subjects. This study was approved by the Ethics Committee of Tokyo Medical University (approval no. SH3298). 
A total of 30 patients were enrolled after obtaining written informed consent at the Department of Obstetrics and Gynecology, Tokyo Medical University between March 2017 and May 2018. This included 12 patients with endometrial adenocarcinoma who underwent total hysterectomy or pelvic and para-aortic lymphadenectomy simultaneously. Cancer tissue samples were collected during the operation. Table I lists the characteristics of cancer patients. Control endometrial specimens were collected from 18 patients with benign gynecological diseases who underwent hysterectomy. For the control endometrium, only patients with proliferative phase of the menstrual cycle (also recognized as the follicular phase in the ovarian cycle) were enrolled since stromal cells differentially express KLF5 depending on decidualization (15), resulting in large variations in gene expression. There were no statistical differences in age or body mass index between the cancer patients and controls. After surgical removal, tissues were trimmed and immediately stored at $-80^{\circ} \mathrm{C}$ until further use for mRNA and protein extraction. For morphological analyses, a part of the samples were fixed in $4 \%$ paraformaldehyde and embedded in paraffin.

Cell culture. Human endometrial cancer cell lines, Ishikawa and HEC1, were kindly provided by Dr Masato Nishida from the Department of Obstetrics and Gynecology, National Kasumigaura Hospital (Tsuchiura, Japan) and procured from the National Institute of Health Sciences (Tokyo, Japan), respectively. Cells were cultured in Dulbecco's modified Eagle's medium (Thermo Fisher Scientific, Inc.) containing 10\% fetal bovine serum (Thermo Fisher Scientific, Inc.), $100 \mathrm{U} / \mathrm{ml}$ penicillin, and $100 \mu \mathrm{g} / \mathrm{ml}$ streptomycin (Thermo Fisher Scientific, Inc.) at $37^{\circ} \mathrm{C}$ in a $5 \% \mathrm{CO}_{2}$ and $95 \%$ air humidified incubator. Cells were periodically checked for mycoplasma infection using MycoAlert Mycoplasma Detection Kit (Lonza). They were cultured with primocin (Invivogen; Thermo Fisher Scientific, Inc.) to inhibit mycoplasma infection during the regular maintenance, but not during the experiments.

KLF5 knockdown. Table II shows the sequence of two different shRNA sequences used to silence KLF5. The pLKO.1 construct (Addgene, Inc.) with the shRNA sequence and lentiviral packaging plasmids (Addgene, Inc.) were used to produce lentiviral particles for the transduction of shRNA-mediated knockdown system as previously reported (16). The pLKO.1-shSCR, containing scrambled shRNA which is not expected to target any mRNA, was used as the control (Addgene, Inc.). Cells were exposed to the lentiviral particles and transduced cells were selected using $3 \mu \mathrm{g} / \mathrm{ml}$ puromycin dihydrochloride (Nacalai Tesque) for two days. Samples for mRNA and protein analyses were collected after an additional day of culture without puromycin.

mRNA expression analysis. Total RNA was extracted using ISOGEN (\#311-02501; Nippon Gene) and reverse-transcribed using Superscript III Reverse Transcriptase (\#1808044; Thermo Fisher Scientific, Inc.) according to the manufacturer's instructions. Reverse transcription-quantitative PCR (RT-qPCR) was performed using the Power SYBR-Green Master Mix (\#4368577; Thermo Fisher Scientific, Inc.) and Applied Biosystems StepOnePlus ${ }^{\mathrm{TM}}$ Real-Time PCR system
(Thermo Fisher Scientific, Inc.). Data were normalized to the endogenous control, 18S rRNA, and they were analyzed using the $2^{-\Delta \Delta \mathrm{Cq}}$ method (17). Table II lists the primer sequences used in RT-qPCR.

Western blotting. Proteins were extracted by Pierce RIPA buffer (Thermo Fisher Scientific, Inc.) and the concentrations were measured using Pierce BCA protein assay kit (Thermo Fisher Scientific, Inc.). Thirty microgram each of proteins were, then, denatured by NuPAGE ${ }^{\mathrm{TM}}$ LDS Sample Buffer, separated by NuPAGE ${ }^{\circledR}$ Bis-Tris gel electrophoresis (4-12\% gradient; Thermo Fisher Scientific, Inc.), and transferred to polyvinylidene fluoride membranes using the iBlot Dry Blotting System (Thermo Fisher Scientific, Inc.). After blocking with 5\% skim milk in TBST for $1 \mathrm{~h}$ at room temperature, the membrane was incubated with anti-KLF5 antibody (1:1,000 dilution, \#ab137676; Abcam) at $4^{\circ} \mathrm{C}$ overnight followed by horseradish peroxidase-conjugated secondary antibody (\#7074; Cell Signaling Technology) at room temperature for $2 \mathrm{~h}$. Signal from the protein bands were visualized using the ECL western blotting detection system (Bio-Rad Laboratories). $\beta$-actin (1:10,000 dilution, \#MB1051R; Merck) was used as the loading control.

Immunohistochemistry. Paraffin-embedded samples were sectioned, deparaffinized, antigens retrieved using heat-induced retrieval, and incubated with the anti-KLF5 antibody (1:100 dilution). The signal from the protein was visualized using the diaminobenzidine staining kit (Nichirei Bioscience, Inc.). Hematoxylin was used to stain the nuclei.

Cell proliferation and apoptosis assays. Cell proliferation was measured by the MTT assay using CellTiter $96^{\circledR}$ Non-Radioactive Cell Proliferation Assay kit (Promega), and cell apoptosis was analyzed by TUNEL assay using in situ cell death detection kit (Roche Diagnostics) following the manufacturer's instructions. Cells, treated with DNase I (100 U/ml; Sigma-Aldrich; Merck KGaA) to artificially induce the DNA fragmentation, was used as a positive control for TUNEL assay.

Statistical analysis. Data were represented as mean \pm standard error of the mean. Differences between groups were analyzed using the unpaired Student's t-test and differences among three groups were evaluated with ANOVA following Tukey's post hoc test by using SPSS software. $\mathrm{P}<0.05$ was considered statistically significant.

\section{Results}

KLF5 is upregulated in endometrial cancer. Thirty patients, including 12 patients with endometrial cancer and 18 with benign gynecological diseases (controls), were enrolled. We used RT-qPCR and western blotting to demonstrate increased mRNA and protein levels of KLF5, respectively, in the endometrial cancer samples than those in the control endometrium (Fig. 1A and B). Immunohistochemistry showed moderate and faint expression in glandular epithelial and stromal cells, respectively, in the control endometrium, while a strong signal was observed in tumor cells in the endometrial cancer specimens (Fig. 1C). 
Table I. Characteristics of patients with endometrial cancer.

\begin{tabular}{|c|c|c|c|c|c|c|}
\hline Patient & $\begin{array}{l}\text { Age, } \\
\text { years }\end{array}$ & $\begin{array}{l}\text { Body mass } \\
\text { index }\end{array}$ & Histology & $\begin{array}{l}\text { Stage } \\
(\mathrm{pT})\end{array}$ & $\begin{array}{l}\text { Lymph node } \\
\text { metastasis }(\mathrm{pN})\end{array}$ & $\begin{array}{l}\text { Histological } \\
\text { grade }\end{array}$ \\
\hline Case 1 & 40 & 17.4 & $\begin{array}{l}\text { Endometrioid adenocarcinoma with squamous } \\
\text { differentiation }\end{array}$ & pT1a & No & G1 \\
\hline Case 2 & 76 & 23.8 & Mixed endometrioid undifferentiated carcinoma & pT1b & No & G3 \\
\hline Case 3 & 58 & 25.0 & Endometrioid adenocarcinoma & pT1a & No & G1 \\
\hline Case 4 & 71 & 27.9 & Endometrioid adenocarcinoma & pT1a & No & G1 \\
\hline Case 5 & 53 & 34.9 & $\begin{array}{l}\text { Endometrioid adenocarcinoma with undifferentiated } \\
\text { carcinoma }\end{array}$ & pT1a & No & G3 \\
\hline Case 6 & 82 & 16.9 & $\begin{array}{l}\text { Endometrioid adenocarcinoma with undifferentiated } \\
\text { carcinoma }\end{array}$ & pT1a & No & G1 \\
\hline Case 7 & 52 & 19.5 & Endometrioid adenocarcinoma & pT1a & No & G1 \\
\hline Case 8 & 52 & 32.1 & Endometrioid adenocarcinoma & pT1a & N1 & G1 \\
\hline Case 9 & 66 & 26.1 & Endometrioid adenocarcinoma & pT1a & N1 & G1 \\
\hline Case 10 & 52 & 24.2 & Endometrioid adenocarcinoma & pT2 & N1 & G1 \\
\hline Case 11 & 57 & 25.6 & Endometrioid adenocarcinoma & pT1a & NX & G1 \\
\hline Case 12 & 58 & 23.0 & Endometrioid adenocarcinoma & pT1b & N1 & G1 \\
\hline
\end{tabular}

Table II. Specific shRNA and primer sequences.

\begin{tabular}{ll}
\hline Target gene & \multicolumn{1}{c}{ Sequence (5'-3') } \\
\hline shRNAs & \\
SCR (control) & CCTAAGGTTAAGTCGCCCTCG \\
KLF5 sh1 & GCTGTAATGTATATGGCTTTA \\
KLF5 sh2 & CCTATAATTCCAGAGCATAAA \\
Primers & \\
18S rRNA-F & GCAATTATTCCCCATGAACG \\
18S rRNA-R & GGCCTCACTAAACCATCCAA \\
KLF5-F & AACGACGCATCCACTACTGC \\
KLF5-R & CAGCCTTCCCAGGTACACTT \\
CCNA2-F & CACCATTCATGTGGATGAAGC \\
CCNA2-R & ACACTCACTGGCTTTTCATCTT \\
CCNB1-F & GCAGCAGGAGCTTTTGCTT \\
CCNB1-R & CCAGGTGCTGCATAACTGGA \\
CCND1-F & CAATGACCCCGCACGATTTC \\
CCND1-R & AAGTTGTTGGGGCTCCTCAG \\
CCNE1-F & CAGCCCCATCATGCCGAG \\
CCNE1-R & TTGTCAGGTGTGGGGATCAG \\
\hline
\end{tabular}

CCN, cyclin; F, forward; R, reverse; KLF5, Krüppel-like factor 5; SCR, scramble; sh/shRNA, short hairpin RNA.

Silencing of KLF5 inhibits endometrial cancer cell proliferation. To investigate the role of KLF5 in endometrial cancer, Ishikawa cells were transduced with the lentiviral constructs bearing two different shRNAs targeting KLF5 and subjected to loss-of-function assays. KLF5 knockdown efficiency was successfully validated in the transduced Ishikawa cells (Fig. 2A and B). KLF5 expression is upregulated in the cancer cells, and it possibly supports the oncogenic processes, such as proliferation and

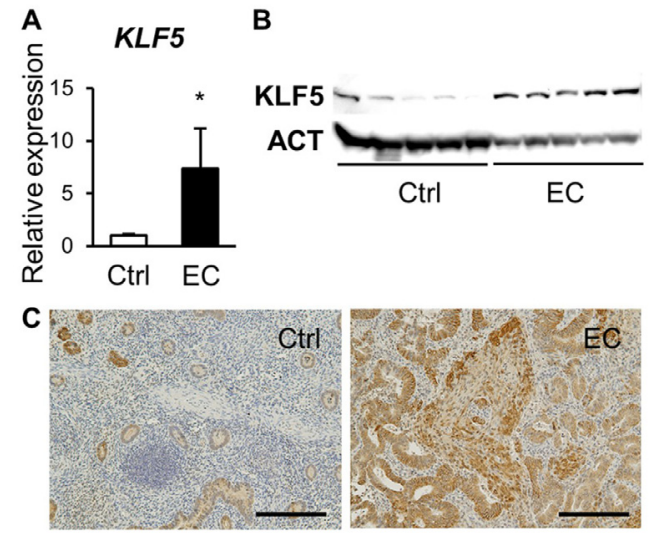

Figure 1. Upregulation of KLF5 expression in EC. (A) mRNA and (B and C) protein expression levels of KLF5 were analyzed by (A) reverse transcription-quantitative PCR, (B) western blotting and (C) immunohistochemistry in Ctrl and EC tissues. Data are presented as the mean \pm standard error of the mean. Scale bar, $200 \mu \mathrm{m} .{ }^{*} \mathrm{P}<0.05$ statistical significance compared with control. ACT, $\beta$-actin; Ctrl, control endometrium; EC, endometrial cancer; KLF5, Krüppel-like factor 5.

apoptosis. The results from the MTT assays revealed that depletion of KLF5 reduced cell proliferation (Fig. 2C). To confirm the role of KLF5 in cell proliferation, HEC1 cells were transduced with the shRNA targeting KLF5 and subjected to MTT assays. As observed in Ishikawa cells, KLF5 knockdown also suppressed the proliferation of HEC1 cells (Fig. 2D).

Transactivation of the cyclins' expression by KLF5 is believed to enhance the cell proliferation (8). Among the cyclins (CCN), the expression of CCNE1 was significantly downregulated in the KLF5-depleted cells (Fig. 2E). To further validate the role of KLF5 in tumorigenesis, we analyzed the cell survival potential. Cell apoptosis was absent in the KLF5-depleted cells, as seen from the TUNEL assays, indicating that the reduction in the number of cells was not due to cell death (Fig. 2F). 

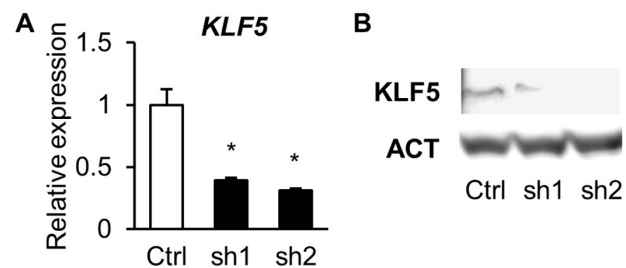

Ctrl sh1 sh2
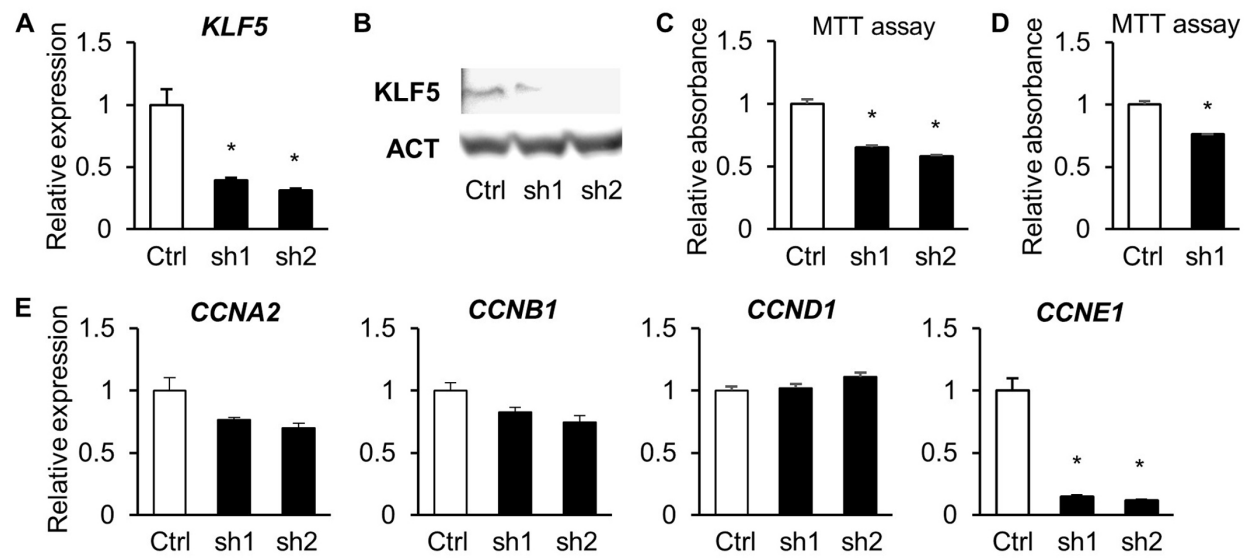

$\mathbf{F}$

Ctrl
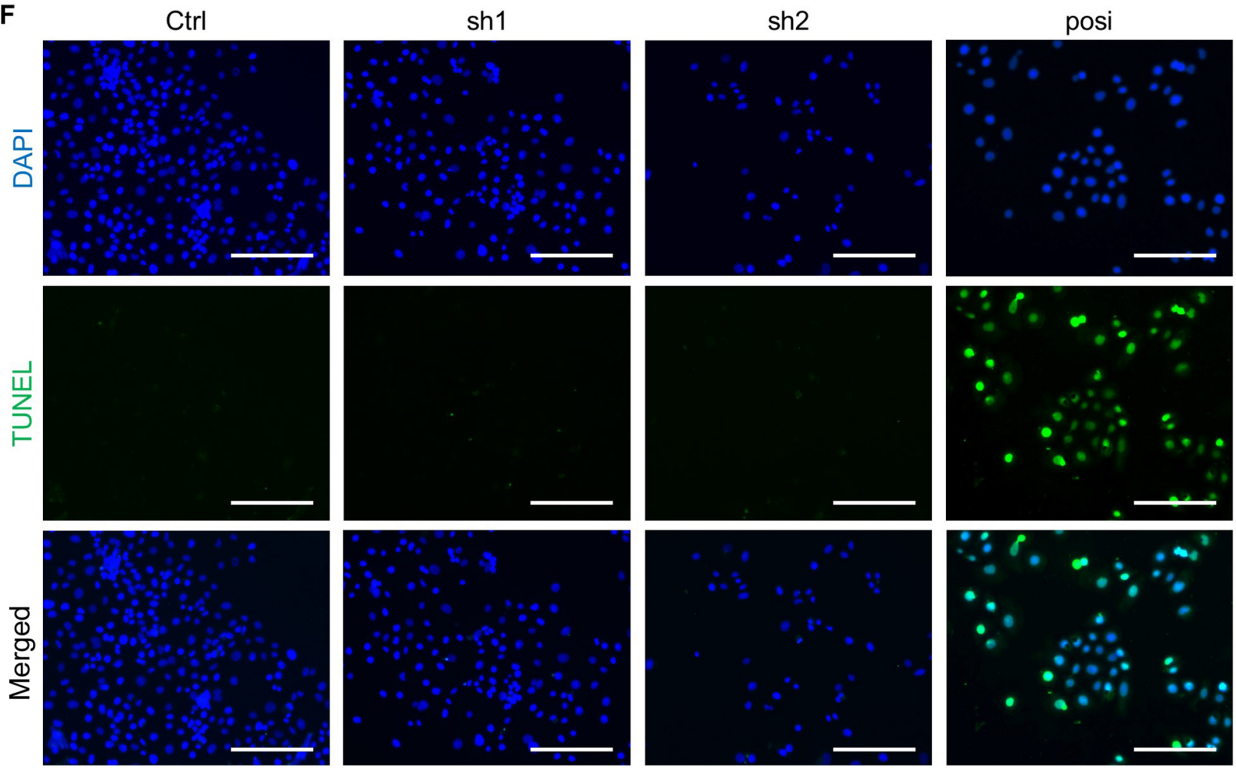

Figure 2. KLF5 knockdown inhibits cell proliferation but does not induce apoptosis. (A and B) Ishikawa cells were depleted of KLF5 upon the transduction of two different shRNAs (sh1 and sh2) targeting KLF5. Non-targeting scramble shRNA was used as a Ctrl. Knockdown efficiency was measured by assessing (A) mRNA and (B) protein expression levels. (C) Cell proliferation was measured using MTT assays. (D) HEC1 cells were depleted of KLF5 and cell proliferation was determined using MTT assays. (E) mRNA expression levels of CCNA2, CCNB1, CCND1 and CCNE1 were analyzed using reverse transcription-quantitative PCR. (F) Cell apoptosis was analyzed using a TUNEL assay. The far right panel represents the posi. Scale bar, $200 \mu \mathrm{m}$. Data are presented as the mean \pm standard error of the mean. ${ }^{*} \mathrm{P}<0.05$ statistical significance compared with control. ACT, $\beta$-actin; $\mathrm{CCN}$, cyclin; Ctrl, control; KLF5, Krüppel-like factor 5; posi, positive control; sh/shRNA, short hairpin RNA.

\section{Discussion}

Endometrial cancer is the most common cancer of the reproductive system of females in developed countries whose incidence has been increasing worldwide $(1,2)$. The treatment options currently available for endometrial cancer are limited: Surgical removal is preferentially recommended. However, in cases of the patients with physiological complications or those who are very old, radiation might be the next choice. The pathogenesis and pathophysiology of endometrial cancer remains to be understood fully. Exploring the molecular mechanism(s) underlying tumorigenesis and metastasis of endometrial cancer will help identify new therapeutic strategies for patients. Recent studies have reported that KLF5 regulates cancer phenotypes, including cellular proliferation, apoptosis, differentiation, and motility (6-8). KLF5 has a dual role in being a tumor suppressor or oncogene, depending on the cellular and genetic environments (8). Thus, the role of KLF5 differs and needs to be studied in each cancer type.
The molecular mechanism underlying the oncogenic role of KLF5 has been clearly elucidated in colorectal cancer. More than $50 \%$ of colon cancers exhibit activating mutations in the oncogene K-RAS; this upregulates KLF5 and promotes cell proliferation (9). KLF5 is also a critical mediator of the oncogene H-RAS $(18,19)$ and tumor suppressor APC $(20,21)$. Increases the expression of KLF5 is associated with poor prognosis (22). In vitro knockdown experiments have shown that KLF5 interacts with b-catenin and localizes to the nucleus where the complex transactivates target genes, such as CCND1 and c-MYC (20,22). Genome-wide association studies identified the regulatory region of KLF5 as a risk locus for endometrial cancer (23). However, the expression and function of KLF5 in endometrial cancer remains to be explored. This study showed the upregulation of KLF5 in endometrial cancer, and the shRNA-mediated depletion of KLF5 inhibited Ishikawa and HEC1 cell proliferation. In addition, we have demonstrated that the depletion of KLF5 downregulated the expression of CCNE1, but not that of CCND1. It has been 
reported that KLF5 binds to the first intron of CCNE1 and it regulates transcription and cellular proliferation in bladder cancer (24). Therefore, KLF5 promotes cell proliferation by trans-activating a variety of cyclins depending on the cancer type and cellular microenvironments. CCNE1 expression is associated with the proliferative and aggressive potential, and it is an indicator in the prognosis for endometrial cancer $(25,26)$. The role of CCNE1 within KLF5-mediated cell proliferation is not apparent in this study; however, KLF5 regulates cell proliferation, at least in part, through the action of CCNE1. Attempting to rescue the KLF5 compromised cell proliferation with CCNE1 could be informative. The exact mechanism of KLF5 regulation of target genes remains to be explored. SUMOyration regulates KLF5 nuclear localization, and phosphorylation enhances KLF5 transactivation $(27,28)$. The analyses of nuclear localization, protein activation, and/or promoter activity could further confirm the role of KLF5 in transcriptional regulation.

KLF5 has also been reported to modulate cell survival by inhibiting apoptosis. KLF5 binds to the core promoter region and induces the expression of the anti-apoptotic protein survivin in leukemia (29). In colon cancer HCT116 cells, KLF5 induces the expression of the survival kinase Pim1 that phosphorylates and inactivates the pro-apoptotic protein BAD (30). In this study, however, silencing KLF5 decreased cell numbers but did not induce apoptosis in endometrial cancer cells, suggesting that KLF5 acts as an oncogene in endometrial cancer by directly promoting cell proliferation rather than inducing cell survival.

This study has some limitations. First, we used a small sample size. This led to a large variation in the expression of KLF5 in the endometrial cancer samples. This could also be attributed to the stage-dependent change in KLF5 expression in non-tumor cells. KLF5 is expressed in normal endometrial epithelial cells (14) and stromal cells, especially after decidualization (15). Therefore, the proportion of tumor cells against normal cells generates a difference in gene expression. Nevertheless, we found a statistical difference in KLF5 expression, especially protein levels, in the endometrial cancer specimens. KLF5 undergoes regulation at the transcriptional, post-transcriptional, and post-translational stages, including its protein stability induced by differential proteasomal degradation (31-34). This could have resulted in the clear and strong signals of KLF5 protein in the endometrial cancer specimens as analyzed by western blotting and immunohistochemistry. However, a large-scale study of KLF5 expression in the endometrial cancer, and its correlation with the treatment outcomes and long-time prognosis could generate valuable information. The second limitation is that only early phase cancer patients were enrolled in this study. KLF5 expression might vary across the different stages and grades of cancer progression. We have focused on the proliferative role of KLF5 and, therefore, investigated the early phase tumor. The KLF5 expression in aggressive tumors as well as its function in tumor metastasis is of interest for the next studies.

In conclusion, we have demonstrated the upregulation of KLF5 in endometrial cancer. Furthermore, KLF5 regulates cell proliferation, at least in part, through the action of CCNE1. Future experiments should also focus on the role of KLF5 in epithelial-mesenchymal transition and/or metastasis in endometrial cancer. Large-scale and randomized clinical studies will further help validate the potential of KLF5 as a therapeutic target for endometrial cancer.

\section{Acknowledgements}

Not applicable.

\section{Funding}

The present study was supported by the Japan Society for the Promotion of Science (grant no. 19K09835) and International Joint Usage/Research Center, Institute of Medical Science, University of Tokyo.

\section{Availability of data and materials}

The datasets used and/or analyzed during the current study are available from the corresponding author on reasonable request.

\section{Authors' contributions}

TM, JK and KK wrote the manuscript, analyzed the data and performed the experiments. TM and JK reviewed the raw data and confirmed its authenticity. TM, JK, FT, KI and HN collected the samples. KK, FT, KI and HN designed the study. All authors read and approved the final manuscript.

\section{Ethics approval and consent to participate}

The present study was approved by the Ethics Committee of Tokyo Medical University (Tokyo, Japan). Written informed consent was obtained from the patients or guardians.

\section{Patient consent for publication}

Not applicable.

\section{Competing interests}

The authors declare that they have no competing interests.

\section{References}

1. Felix AS and Brinton LA: Cancer progress and priorities: Uterine cancer. Cancer Epidemiol Biomarkers Prev 27: 985-994, 2018.

2. Bray F, Ferlay J, Soerjomataram I, Siegel RL, Torre LA and Jemal A: Global cancer statistics 2018: GLOBOCAN estimates of incidence and mortality worldwide for 36 cancers in 185 countries. CA Cancer J Clin 68: 394-424, 2018.

3. Morice P, Leary A, Creutzberg C, Abu-Rustum N and Darai E: Endometrial cancer. Lancet 387: 1094-1108, 2016.

4. Sheikh MA, Althouse AD, Freese KE, Soisson S, Edwards RP Welburn S, Sukumvanich P, Comerci J, Kelley J, LaPorte RE and Linkov F: USA endometrial cancer projections to 2030: Should we be concerned? Future Oncol 10: 2561-2568, 2014.

5. McAlpine JN, Temkin SM and Mackay HJ: Endometrial cancer: Not your grandmother's cancer. Cancer 122: 2787-2798, 2016.

6. Simmen RC, Pabona JM, Velarde MC, Simmons C, Rahal O and Simmen FA: The emerging role of Krüppel-like factors in endocrine-responsive cancers of female reproductive tissues. J Endocrinol 204: 223-231, 2010.

7. Simmen RCM, Heard ME, Simmen AM, Montales MT, Marji M, Scanlon S and Pabona JM: The Krüppel-like factors in female reproductive system pathologies. J Mol Endocrinol 54: R89-R101, 2015. 
8. Diakiw SM, D'Andrea RJ and Brown AL: The double life of KLF5: Opposing roles in regulation of gene-expression, cellular function, and transformation. IUBMB Life 65: 999-1011, 2013

9. Nandan MO, McConnell BB, Ghaleb AM, Bialkowska AB, Sheng H, Shao J, Babbin BA, Robine S and Yang VW: Krüppel-like factor 5 mediates cellular transformation during oncogenic KRAS-induced intestinal tumorigenesis. Gastroenterology 134: 120-130, 2008.

10. Marrero-Rodríguez D, Taniguchi-Ponciano K, Jimenez-Vega F, Romero-Morelos P, Mendoza-Rodríguez M, Mantilla A, RodriguezEsquivel M, Hernandez D, Hernandez A, Gomez-Gutierrez G, et al: Krüppel-like factor 5 as potential molecular marker in cervical cancer and the KLF family profile expression. Tumor Biol 35 $11399-11407,2014$

11. Zheng HQ, Zhou Z, Huang J, Chaudhury L, Dong JT and Chen C: Krüppel-like factor 5 promotes breast cell proliferation partially through upregulating the transcription of fibroblast growth factor binding protein 1 . Oncogene 28: 3702-3713, 2009.

12. Meyer SE, Hasenstein JR, Baktula A, Velu CS, Xu Y, Wan H, Whitsett JA, Gilks CB and Grimes HL: Kruppel-like factor 5 is not required for K-RasG12D lung tumorigenesis, but represses ABCG2 expression and is associated with better disease-specific survival. Am J Pathol 177: 1503-1513, 2010.

13. Chen C, Bhalala HV, Vessella RL and Dong JT: KLF5 is frequently deleted and down-regulated but rarely mutated in prostate cancer. Prostate 55: 81-88, 2003.

14. Shi H, Zhang Z, Wang X, Liu S and Teng CT: Isolation and characterization of a gene encoding human Kruppel-like factor 5 (IKLF): Binding to the CAAT/GT box of the mouse lactoferrin gene promoter. Nucleic Acids Res 27: 4807-4815, 1999.

15. Sun X, Zhang L, Xie H, Wan H, Magella B, Whitsett JA and Dey SK: Kruppel-like factor 5 (KLF5) is critical for conferring uterine receptivity to implantation. Proc Natl Acad Sci USA 109: $1145-1150,2012$

16. Stewart SA, Dykxhoorn DM, Palliser D, Mizuno H, Yu EY An DS, Sabatini DM, Chen ISY, Hahn WC, Sharp AP, et al: Lentivirus-delivered stable gene silencing by RNAi in primary cells. RNA 9: 493-501, 2003.

17. Livak KJ and Schmittgen TD: Analysis of relative gene expression data using real-time quantitative PCR and the 2(-Delta Delta C(T)) method. Methods 25: 402-408, 2001.

18. Nandan MO, Chanchevalap S, Dalton WB and Yang VW: Krüppel-like factor 5 promotes mitosis by activating the cyclin $\mathrm{B} 1 / \mathrm{Cdc} 2$ complex during oncogenic Ras-mediated transformation. FEBS Lett 579: 4757-4762, 2005.

19. Nandan MO, Yoon HS, Zhao W, Ouko LA, Chanchevalap S and Yang VW: Krüppel-like factor 5 mediates the transforming activity of oncogenic H-Ras. Oncogene 23: 3404-3413, 2004.

20. McConnell BB, Bialkowska AB, Nandan MO, Ghaleb AM Gordon FJ and Yang VW: Haploinsufficiency of Krüppel-like factor 5 rescues the tumor-initiating effect of the Apc(Min) mutation in the intestine. Cancer Res 69: 4125-4133, 2009.

21. Nandan MO, Ghaleb AM, McConnell BB, Patel NV, Robine S and Yang VW: Krüppel-like factor 5 is a crucial mediator of intestinal tumorigenesis in mice harboring combined ApcMin and KRASV12 mutations. Mol Cancer 9: 63, 2010.
22. Takagi Y, Sakai N, Yoshitomi H, Furukawa K, Takayashiki T, Kuboki S, Takano S, Suzuki D, Kagawa S, Mishimi T, et al: High expression of Krüppel-like factor 5 is associated with poor prognosis in patients with colorectal cancer. Cancer Sci 111: 2078-2092, 2020.

23. Cheng TH, Thompson DJ, O'Mara TA, Painter JN, Glubb DM, Flach S, Lewis A, French JD, Freeman-Mills L, Church D, et al: Five endometrial cancer risk loci identified through genome-wide association analysis. Nat Genet 48: 667-674, 2016.

24. Pattison JM, Posternak V and Cole MD: Transcription factor KLF5 binds a cyclin E1 polymorphic intronic enhancer to confer increased bladder cancer risk. Mol Cancer Res 14: 1078-1086, 2016.

25. Nakayama K, Rahman MT, Rahman M, Nakamura K, Ishikawa M, Katagiri H, Sato E, Ishibashi T, Iida K, Ishikawa N and Kyo S: CCNE1 amplification is associated with aggressive potential in endometrioid endometrial carcinomas. Int $\mathrm{J}$ Oncol 48: 506-516, 2016.

26. Santala S, Talvensaari-Mattila A, Soini Y and Santala M: Cyclin E expression correlates with cancer-specific survival in endometrial endometrioid adenocarcinoma. Anticancer Res 35: 3393-3397, 2015.

27. Du JX, Bialkowska AB, McConnell BB and Yang VW: SUMOylation regulates nuclear localization of Krüppel-like factor 5. J Biol Chem 283: 31991-32002, 2008.

28. Zhang $\mathrm{Z}$ and Teng CT: Phosphorylation of Kruppel-like factor 5 (KLF5/IKLF) at the CBP interaction region enhances its transactivation function. Nucleic Acids Res 31: 2196-2208, 2003.

29. Zhu N, Gu L, Findley HW, Chen C, Dong JT, Yang L and Zhou M KLF5 interacts with p53 in regulating survivin expression in acute lymphoblastic leukemia. J Biol Chem 281: 14711-14718, 2006.

30. Zhao Y, Hamza MS, Leong HS, Lim CB, Pan YF, Cheung E, Soo KC and Iyer NG: Kruppel-like factor 5 modulates p53-independent apoptosis through Pim1 survival kinase in cancer cells. Oncogene 27: 1-8, 2008

31. Chen C, Zhou Z, Guo P and Dong JT: Proteasomal degradation of the KLF5 transcription factor through a ubiquitin-independent pathway. FEBS Lett 581: 1124-1130, 2007.

32. Liu N, Li H, Li S, Shen M, Xiao N, Chen Y, Wang Y, Wang W, Wang R, Wang Q, et al: The Fbw7/human CDC4 tumor suppressor targets proproliferative factor KLF5 for ubiquitination and degradation through multiple phosphodegron motifs. J Biol Chem 285: 18858-18867, 2010 .

33. Zhao D, Zheng HQ, Zhou Z and Chen C: The Fbw7 tumor suppressor targets KLF5 for ubiquitin-mediated degradation and suppresses breast cell proliferation. Cancer Res 70: 4728-4738, 2010.

34. Zhao D, Zhi X, Zhou Z and Chen C: TAZ antagonizes the WWP1-mediated KLF5 degradation and promotes breast cell proliferation and tumorigenesis. Carcinogenesis 33: 59-67, 2012.

This work is licensed under a Creative Commons Attribution-NonCommercial-NoDerivatives 4.0 International (CC BY-NC-ND 4.0) License. 